\title{
RSV Mediates Pseudomonas aeruginosa Binding to Cystic Fibrosis and Normal Epithelial Cells
}

\author{
BART E. VAN EWIJK, TOM F.W. WOLFS, PIET C. AERTS, KOK P.M. VAN KESSEL, ANDRE FLEER, JAN L.L. KIMPEN, \\ AND CORNELIS K. VAN DER ENT
}

\author{
Cystic Fibrosis Centre and Department of Pediatric Respiratory Medicine [B.E.E., C.K.E.]; Department of Pediatric Infectious Diseases \\ [T.R.W.W., P.C.A., A.F., J.L.L.K.]; Eijkman Winkler Institute [B.E.E., P.C.A., K.P.M.K.]; Wilhelmina Children's Hospital, University \\ Medical Centre Utrecht, 3508 AB Utrecht, The Netherlands
}

\begin{abstract}
Cystic fibrosis lung disease typically has a course of exacerbations and remissions, suggesting that external factors like viral infections can influence this course. Clinical data suggest synergism between respiratory syncytial virus (RSV) infections and Pseudomonas aeruginosa in cystic fibrosis (CF) lung disease. We studied the influence of RSV infection on adherence of $P$. aeruginosa to IB3-1, HEp-2, and A549 epithelial cell monolayers in vitro. RSV infection of epithelial cells as well as simultaneous addition of RSV and $P$. aeruginosa to noninfected cells both strongly enhanced the pseudomonal adherence to epithelial cells. The increased adherence varied from 1.2- to 8.2-fold in case of previous RSV infection, and from 1.7- to 16.1-fold in case of simultaneous addition. We observed direct binding of RSV to $P$. aeruginosa, and blocking of RSV with heparin eliminated the effect on increased adherence. This suggests that RSV possibly acts as a coupling agent between $P$. aeruginosa and epithelial cells. In conclusion, RSV enhances $P$. aeruginosa infection of respiratory epithelial cells. It suggests a role of specific viral-bacterial interactions in exacerbations of $\mathrm{CF}$ lung disease, which could have important implications on prevention and treatment. (Pediatr Res 61: 398-403, 2007)
\end{abstract}

$\mathrm{C}^{\mathrm{F}}$ is characterized by chronic inflammation, bacterial colonization, and recurrent infections of the lung, which results in irreversible deterioration of lung function and early death. Pseudomonas aeruginosa is one of the most important bacterial pathogens in CF. Chronic infection of the airways with $P$. aeruginosa accelerates the progression to irreversible lung damage (1). Impaired mucociliary transport and antipseudomonal defense, specific binding to asialoGM-1 receptors, and persistent inflammation probably play a role in chronic pseudomonal colonization in CF patients (2). Besides a constitutive inflammatory state, $\mathrm{CF}$ lung disease typically has a course of exacerbations and remissions. This suggests that external factors influence this course, e.g. viral infections. Although the prevalence of viral infections seems to be equal in CF patients and healthy controls $(3,4)$, CF patients are more likely to develop a lower respiratory tract infection with more

Received September 7, 2006; accepted November 15, 2006.

Correspondence: Bart E. van Ewijk, M.D., Cystic Fibrosis Centre and Department of Pediatric Respiratory Medicine, Wilhelmina Children's Hospital, University Medical Centre Utrecht, PO Box 85090 Office KH 01.419.0, 3508 AB Utrecht, Netherlands; e-mail: b.vanewijk@umcutrecht.nl

This work was supported by Wilhelmina Children's Hospital, Research Fund (fellowship to B.E.E.).

DOI: $10.1203 / p d r .0 b 013 e 3180332 d 1 c$ and prolonged symptoms $(3,5,6)$. The mechanisms behind these differences are not elucidated.

Synergism between viruses and bacteria in inducing infections of respiratory epithelium has been described both in vitro (7-9) and in vivo $(10,11)$. Circumstantial evidence suggests that initial pseudomonal colonization and persistent infection in $\mathrm{CF}$ might be facilitated by respiratory viral infections, especially by RSV (12-14). We speculate that synergism between respiratory viral infections and pseudomonal colonization plays an important role in the pathogenesis of $\mathrm{CF}$ pulmonary disease. Therefore, we studied whether RSV infection enhances the adherence of $P$. aeruginosa to human respiratory epithelial cells and we tried to elucidate some of the underlying mechanism.

\section{MATERIALS AND METHODS}

Cell cultures. IB3-1 human cystic fibrosis bronchial cells (JHU-52), HEp-2 human nasopharyngeal carcinoma cells (CCL-23), and A549 human pneumocyte type II carcinoma cells (CCL-185) were obtained from the American Type Culture Collection (ATCC, Manassas, VA). Stocks of cellular suspensions were stored at $-180^{\circ} \mathrm{C}$ in liquid nitrogen. Cells in 24- or 96-well microtiter plates (Costar, Cambridge, $\mathrm{MA}$ ) were cultured in $\mathrm{CO}_{2}$ incubator to confluence at $37^{\circ} \mathrm{C}$ in Iscove's Modified Dulbecco's Medium (IMDM, Invitrogen, Carlsbad, CA) containing 5\% FCS (Hyclone Laboratories, Logan, UT) and gentamicin, $0.01 \mathrm{mg} / \mathrm{mL}$ (Invitrogen). Cells were used for experiments between 10 and 25 passages.

Bacteria and labeling. Two mucoid (Pa01 and $\mathrm{Pa} 02)$ and two nonmucoid (Pa03 and Pa04) clinical P. aeruginosa strains were collected from different CF patients. Two other strains were obtained from ATCC, 39342 (mucoid) and 15692 (nonmucoid). Bacteria were stored in microbanks (Pro-Lab Diagnostics, Austin, TX) at $-70^{\circ} \mathrm{C}$. Before testing, bacteria were grown on blood agar plates (Trypticase Soy agar with 5\% sheep blood, BD Biosciences, San Diego, CA) at $37^{\circ} \mathrm{C}$, inoculated in Todd-Hewitt broth (Difco, Detroit, MI) supplemented with $0.5 \%$ yeast extract and grown shaken overnight at $37^{\circ} \mathrm{C}$.

Bacteria were harvested by centrifugation at $3270 \times g$. Pelleted bacteria were washed three times with PBS (Cambrex, Verviers, Belgium) and pelleted by centrifugation at $9300 \times g$ for $5 \mathrm{~min}$. For cytometric experiments, bacteria were suspended in $0.1 \mathrm{M}$ sodium carbonate buffer $\mathrm{pH} 9.0$ and adjusted in a spectrophotometer to a concentration of $10^{9}$ bacteria/mL $\left(\mathrm{OD}_{660 \mathrm{~nm}}=1.0\right)$. The bacteria were freshly labeled with a saturated FITC solution $(10 \mathrm{mg} / \mathrm{mL}$ DMSO; Merck, Darmstadt, Germany), to a final concentration of $0.5 \mathrm{mg} / \mathrm{mL}$, and incubated for $1 \mathrm{~h}$ at $4^{\circ} \mathrm{C}$. Efficiency of FITC-coupling as measured in the FACS varied between 76 and $92 \%$ labeled bacteria, depending on the bacterial strain. After washing three times with PBS, bacteria were suspended in $\mathrm{PBS}^{2+} / \mathrm{BSA} 2.5 \%\left(\mathrm{Ca}^{2+} 0.15 \mathrm{mM}, \mathrm{Mg}^{2+} 0.5 \mathrm{mM}\right.$ enriched with $2.5 \%$ BSA (Instruchemie, Hilversum, Netherlands)) to a final concentration of

Abbreviations: CF, cystic fibrosis; FACS, fluorescence activated cell sorter; RSV, respiratory syncytial virus 
$2.0 \times 10^{8} \mathrm{CFU} / \mathrm{mL}\left(\mathrm{OD}_{660 \mathrm{~nm}}=0.25\right)$ for direct use, or $4.0 \times 10^{8} \mathrm{CFU} / \mathrm{mL}$ $\left(\mathrm{OD}_{660 \mathrm{~nm}}=0.5\right)$ for simultaneous addition with RSV.

Viral stocks. RSV serotype A (ATCC VR 1302) was grown on HEp-2 monolayers as described previously (15). Aliquots containing $3.0 \times 10^{6}$ $\mathrm{PFU} / \mathrm{mL}$ were stored at $-180^{\circ} \mathrm{C}$ in liquid nitrogen.

Adherence assay. Adherence experiments were performed as described previously (15). For RSV infection experiments, monolayers were grown to confluence in 3-4 d; for experiments with simultaneous addition of RSV and $P$. aeruginosa, including heparin blocking and filtration, monolayers were grown to confluence in $2 \mathrm{~d}$. Medium was removed from the cellular monolayers and $50 \mu \mathrm{L}$ of FITC-labeled bacteria were added. After centrifugation at $160 \times g$ (IEC Centra 3C; IEC, Needham Heights, MA) for $10 \mathrm{~min}$, the plates were incubated at room temperature for $30 \mathrm{~min}$. Total fluorescence (TF) was measured by a fluorescence multi-well plate reader (CytoFluor II; PerSeptive Biosystems, Framingham, MA), with excitation at $485 \mathrm{~nm}$ and emission at $530 \mathrm{~nm}$, three readings per well. Subsequently, wells were washed four times with $\mathrm{PBS}^{2+}$ to remove nonadhering $P$. aeruginosa and after addition of $50 \mu \mathrm{L}$ $\mathrm{PBS}^{2+}$ measured again to determine the fluorescence of adhering bacteria (adherence fluorescence $=\mathrm{AF}$ ). The final adherence was calculated as adherence $=\mathrm{AF} / \mathrm{TF} \times 100(\%)$.

Viral infection of monolayers. RSV infection of IB3-1, HEp-2, and A549 monolayers was induced as described previously (15). Cells and serial dilutions ( $\log 3.0-4.8)$ of $50 \mu \mathrm{L}$ of an RSV suspension or IMDM as a control were incubated in 96 -well microtiter plates at $37^{\circ} \mathrm{C}$ in a $\mathrm{CO}_{2}$ incubator. Cytopathological effect (CPE) and percentage of remaining monolayer for the different RSV dilutions were scored under light microscope after each adherence assay.

Binding of RSV to P. aeruginosa. Equal volumes of nonlabeled bacteria $\left(4.0 \times 10^{8} \mathrm{CFU} / \mathrm{mL}\right)$ and RSV suspension $\left(3.0 \times 10^{6} \mathrm{PFU} / \mathrm{mL}\right.$ and $5.0 \times 10^{7}$ $\mathrm{PFU} / \mathrm{mL}$, respectively) or PBS/BSA $0.5 \%$ as control were co-incubated for 30 $\mathrm{min}$ at $37^{\circ} \mathrm{C}$ in an incubator. After washing and centrifugation twice for $5 \mathrm{~min}$ at $9300 \times g, 25 \mu \mathrm{L}$ of FITC-labeled MAb against RSV-glycoprotein F (Imagen Kit for RSV, Glostrop, Denmark) was added and incubated for 30 $\min$ at $4^{\circ} \mathrm{C}$, followed by two washes and suspension in PBS. Fluorescence of 10,000 bacteria was analyzed in a FACScan flow cytometer, expressed as percentage positive bacteria.

Simultaneous addition of RSV and P. aeruginosa. Equal volumes of FITC-labeled bacteria $\left(4.0 \times 10^{8} \mathrm{CFU} / \mathrm{mL}\right)$ and RSV suspension $\left(3.0 \times 10^{6}\right.$ $\mathrm{PFU} / \mathrm{mL}$ ) or PBS/BSA $0.5 \%$ as control were co-incubated for $30 \mathrm{~min}$ at $37^{\circ} \mathrm{C}$ to form a complex. This mixture $(50 \mu \mathrm{L})$ was added to a 2 -d confluent and non-RSV-infected monolayer in a 96-well microtiter plate. These were incubated at room temperature for $30 \mathrm{~min}$ after centrifugation at $160 \times g$ for 10 min. Adherence was measured as described above. To study the effect of heparin, a solution of $100 \mathrm{IE} / \mathrm{mL}$ sodium heparin was added to the monolayers before adding the bacteria. Heparin-treated monolayers were incubated for 30 min at $37^{\circ} \mathrm{C}$ in a $\mathrm{CO}_{2}$ incubator. Then, the heparin solution was removed, bacteria were added, and adherence was measured as described above.

Filtration and Purification of RSV suspension. RSV suspension as originally cultured was spun through a $100-\mathrm{kD}$ nitrocellulose filter (Microcon $100 \mathrm{kD}$ filter; Millipore Corporation, Bedford, MA) and the filtrate without RSV virions was used to repeat the simultaneous addition experiments. Purified RSV was prepared by polyethylene glycol (PEG) precipitation.

Scanning electron microscopy. RSV-infected and noninfected HEp-2 cells were grown to confluent monolayers on fibrinogen-coated glass cover slips in 24-well microtiter plates. We added $50 \mu \mathrm{L}$ unlabeled $P$. aeruginosa $(\mathrm{Pa} 01)=2.0 \times 10^{8}$ bacteria/mL $\left(\mathrm{OD}_{660 \mathrm{~nm}}=0.25\right)$ to the infected and control monolayers, or co-incubated $P$. aeruginosa and RSV suspension to the noninfected monolayers, and incubated for $30 \mathrm{~min}$ after centrifugation (10 $\mathrm{min}$ at $160 \times g$ ). Plates were washed four times with PBS and fixated during $10 \mathrm{~min}$ at room temperature with glutaraldehyde $2 \%$ (Merck) in $0.1 \mathrm{M}$ phosphate buffer, $\mathrm{pH}$ 7.4. Monolayers were dehydrated stepwise with ethanol $80 \%$ for $10 \mathrm{~min}$ at room temperature, followed by ethanol $99.9 \%$ for $10 \mathrm{~min}$ at room temperature. Then, hexamethyldisilazane, $15 \mu \mathrm{L}$ (Sigma-Aldrich Chemie GmbH, Buchs, Switzerland) was added for $10 \mathrm{~min}$ to each specimen. Samples were glued on a specimen mount with carbon-based glue (CCCadhesive, Electron Microscopy Sciences, Hatfield, PA) after drying. Samples were titanium-sputtered (Sputter Coater 208HR, Cressington Scientific Instruments Ltd., Watford, U.K.) and examined with a scanning electron microscope FEI XL30SFEG (Royal Philips Electronics, Eindhoven, The Netherlands).

Statistics. Cytometric fluorescence adherence experiments were performed three times, and in each experiment seven wells per same treatment were used. Results are expressed as mean \pm SEM. Data of RSV-P. aeruginosa coupling and RSV filtration experiments are from one of two representative experiments and expressed as mean $\pm \mathrm{SD}$. Each of these experiments was performed in duplicate. All comparisons were done by $t$ test with statistical program SPSS for Windows 12.0.2 (SPSS Inc., Chicago, IL). A value of $p<$ 0.05 was considered significant for these comparisons.

\section{RESULTS}

Effect of RSV infection on P. aeruginosa adherence. The in vitro adherence of the different $P$. aeruginosa strains to human respiratory epithelial cells with and without RSV infection was tested to evaluate the effect of virus infection on pseudomonal adherence. Adherence of $P$. aeruginosa to noninfected respiratory epithelial cells varied for each strain and cell type. Maximum adherence to IB3-1 cells was found at an RSV infecting dose of $\log 3.35$ on the third day of RSV infection, to HEp-2 and A549 cells on the fourth day at RSV infecting doses of $\log 3.70$ and 3.18, respectively (data not shown). This RSV infection of respiratory epithelial cells consistently resulted in an increased maximum adherence of all $P$. aeruginosa strains after $30 \mathrm{~min}$ incubation to these cells (IB3-1 d 3, HEp-2, and A549 d 4), compared with noninfected epithelial cells (Fig. 1). It varied between the different strains and cell types from 1.2- to 8.2-fold, compared with no RSV. This maximum increased adherence of each Pseudomonas strain after RSV infection, compared with no RSV, was significant for all strains and all cell types $(p<0.05)$, except for strains $\mathrm{Pa} 01$ and $\mathrm{Pa} 04$ on A549 cells. RSV concentrations above $\log 3.35$ for IB3-1, log 3.7 for HEp-2, and log 3.18 for A549 (Fig. 2) resulted in loss of monolayers due to cytopathological effect on d 3 (IB3-1) and d 4 (HEp-2 and A549), with a decrease of total measured Pseudomonas adherence. In addition, we found the enhanced adherence of $P$. aeruginosa to be RSV dose dependent in all three cell types (Fig. 2), on d 3 (IB3-1) and d 4 (HEp-2 and A549), respectively.

Binding of $\boldsymbol{R S V}$ to $\boldsymbol{P}$. aeruginosa. We hypothesized that the increased pseudomonal adherence could be due to binding to virus-induced up-regulated cellular membrane proteins or to viral glycoproteins expressed on the cell membrane. We first tested whether RSV and Pseudomonas bacteria can bind directly to each other. Pseudomonal suspension was compared with a suspension of RSV and $P$. aeruginosa. RSV with a dose of $3.0 \times 10^{6} \mathrm{PFU} / \mathrm{mL}$ bound directly to all $P$. aeruginosa strains, varying from $0.34 \%$ to $1.67 \%$ (mean, $0.94 \pm 0.58 \mathrm{SD}$ ) RSV glycoprotein F-positive bacteria. In addition, an increased RSV dose of $5.0 \times 10^{7} \mathrm{PFU} / \mathrm{mL}$ resulted in a dosedependent and increased binding of RSV and $P$. aeruginosa. It varied from $4.7 \%$ to $9.97 \%$, mean, $6.58 \pm 2.09 \mathrm{SD}$ (data not shown).

Effect of simultaneous addition of RSV and P. aeruginosa. To further explore the role of RSV in increased pseudomonal adherence, we compared the adherence of $P$. aeruginos $a$ alone with $P$. aeruginosa co-incubated with RSV to noninfected epithelial cells. Adherence of $P$. aeruginosa alone to noninfected respiratory epithelial cells grown to confluence in $2 \mathrm{~d}$ varied for each strain and cell type. The co-incubated RSV-P. aeruginosa complex consistently led to an increased adherence of $P$. aeruginosa to the respiratory epithelial cells, compared with $P$. aeruginosa alone (Fig. 3). This increased adherence varied between the different strains and cell types from 1.7- to 16.1-fold and was significant for all strains and in all cell types $(p<0.05)$. 



Figure 1. Adherence of $P$. aeruginosa strains to RSV infected IB3-1 (A), HEp-2 (B), and A549 (C) monolayers (solid bars) compared with noninfected monolayers (open bars), as measured by cytometric fluorescence assay. Data are represented as mean \pm SEM of three experiments; in each experiment, seven wells per treatment were used. $t$ test $(p<0.05)$, *nonsignificant.
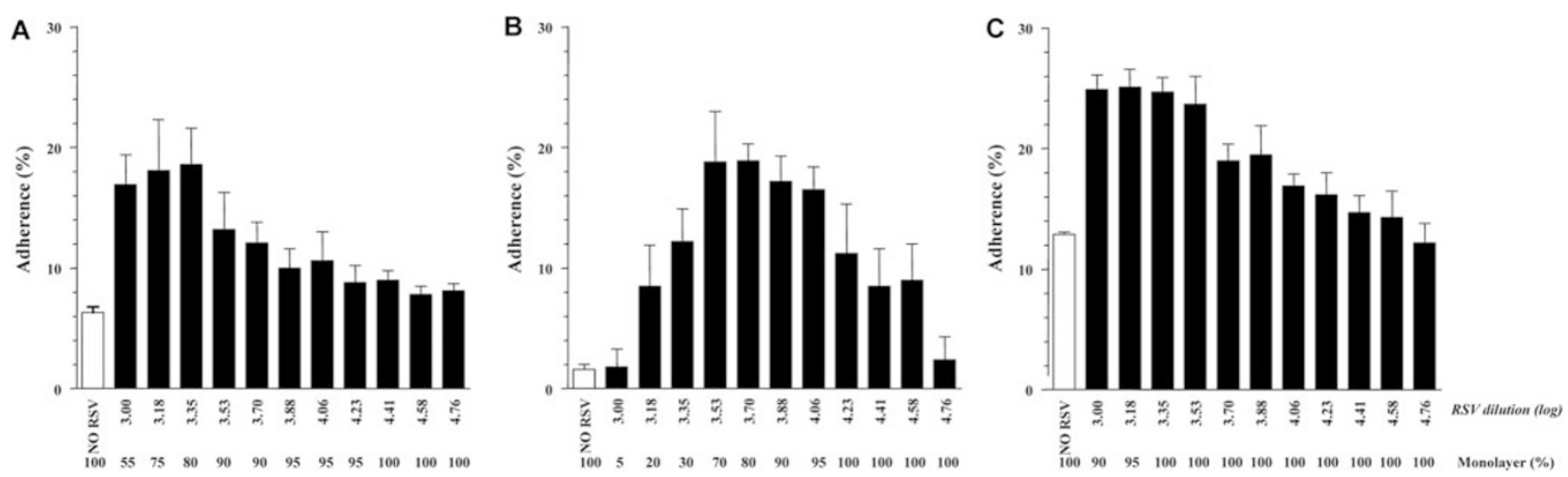

Figure 2. RSV-dose-dependent adherence of $P$. aeruginosa strain Pa01 to noninfected (open bars) and RSV infected IB3-1 (A), HEp-2 (B), and A549 (C) monolayers (solid bars), as measured by cytometric fluorescence assay. Data from one of three representative experiments \pm SD performed in sevenfold are shown.

Effect of RSV filtrate and purified RSV suspension. The increased adherence of $P$. aeruginosa to noninfected epithelial cells when added simultaneously with RSV could be due to RSV acting as a coupling agent between bacteria and epithelial cells or alternatively due to inflammatory mediators in the RSV suspension inducing up-regulation of cellular membrane proteins involved in pseudomonal adherence. To investigate these two possibilities, we incubated $P$. aeruginos $a$ with either unfiltered RSV suspension or RSV filtrate. The RSV filtrate contained all components, including inflammatory mediators from the original RSV culture, except the RSV virions by spinning through a filter. Similar adherence experiments were repeated with a purified RSV suspension without inflammatory mediators. Figure 4 shows that both original and purified RSV suspensions yielded similarly increased pseudomonal adherence of $30.7 \% \pm 0.8 \mathrm{SD}$ and $33.0 \% \pm 1.12 \mathrm{SD}$, respectively. The filtrate induced no increase at all $(3.8 \% \pm 0.5 \mathrm{SD})$, compared with no RSV $(5.6 \% \pm 0.4 \mathrm{SD})$.
Effect of glycoprotein G blocking by heparin on binding. RSV binding to host cells is mediated by glycoprotein G, which can be specifically blocked by heparin. To investigate whether the enhanced binding of the co-incubated RSV $-P$. aeruginosa complex to noninfected epithelial cells was mediated by RSV glycoprotein G, we pretreated cells with heparin. Heparin itself had no effect on the adherence of $P$. aeruginosa to noninfected cells (data not shown). Figure 5 shows that pretreatment of the noninfected cells with heparin led to nearly complete blocking of the enhanced adherence of co-incubated RSV-P. aeruginosa complex, which was significant $(p<0.05)$ for all tested strains. This blocking was heparin dose dependent and similar blocking of enhanced adherence with heparin was found in HEp-2 and A549 cells (data not shown).

Visualization by scanning electron microscopy. Because adherence measured by fluorescence of FITC-labeled bacteria is an indirect and possibly rather insensitive method, we visualized the effect of previous RSV infection and simulta- 

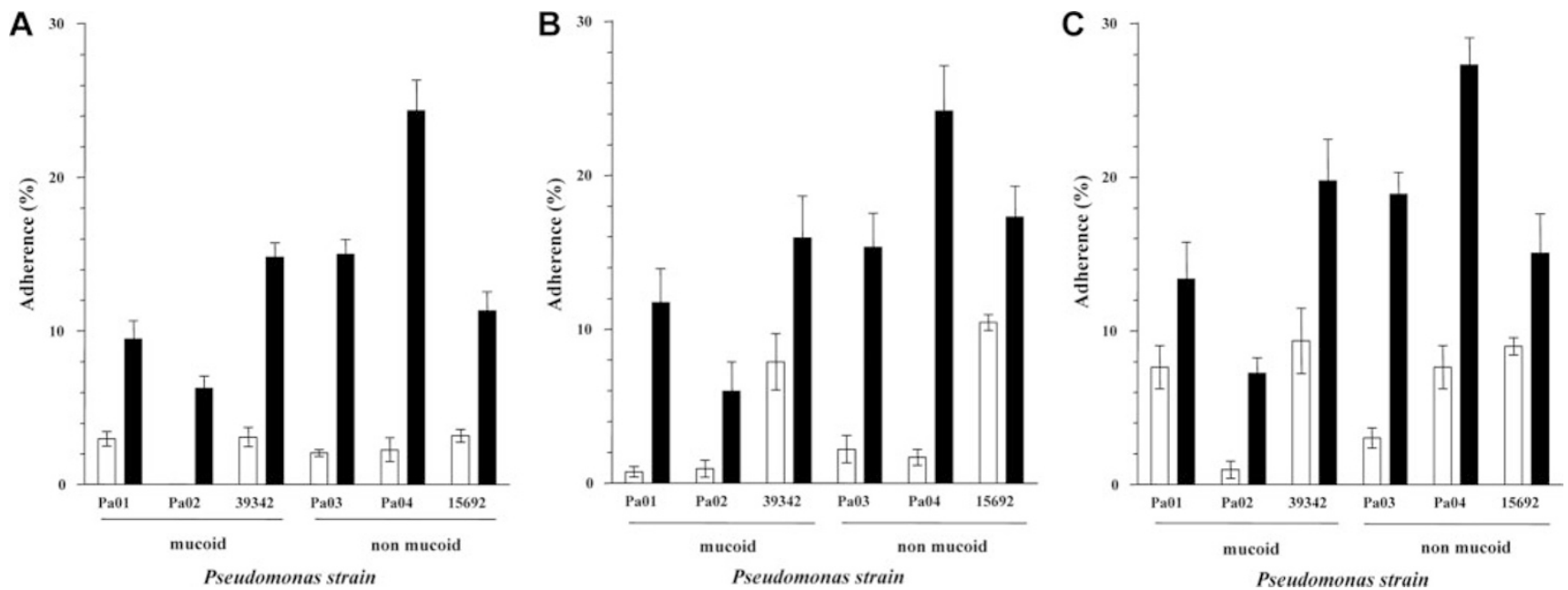

Figure 3. Adherence of $P$. aeruginosa simultaneously added with RSV to noninfected IB3-1 (A), HEp-2 (B), and A549 (C) monolayers (solid bars) compared with $P$. aeruginosa alone (open bars), as measured by cytometric fluorescence assay. Data are represented as mean \pm SEM of three experiments; in each experiment seven wells per treatment were used. Significant difference in all cell lines and with all strains $(t$ test, $p<0.05)$.

neous addition of RSV with $P$. aeruginosa on adherence by performing scanning electron microscopy studies. Incubation of $P$. aeruginosa without RSV showed only a few bacteria adhering to epithelial cells (Fig. 6B). A major increase in adherence was observed when $P$. aeruginosa was added to RSV infected epithelial cells, adhering mainly to syncytia induced by the RSV infection (Fig. 6,C and $D$ ). In contrast, a more even distribution was observed when $P$. aeruginosa and RSV were added simultaneously to uninfected epithelial cells, again considerably increased compared with adherence in the absence of RSV (Fig. 6,E and $F$ ).

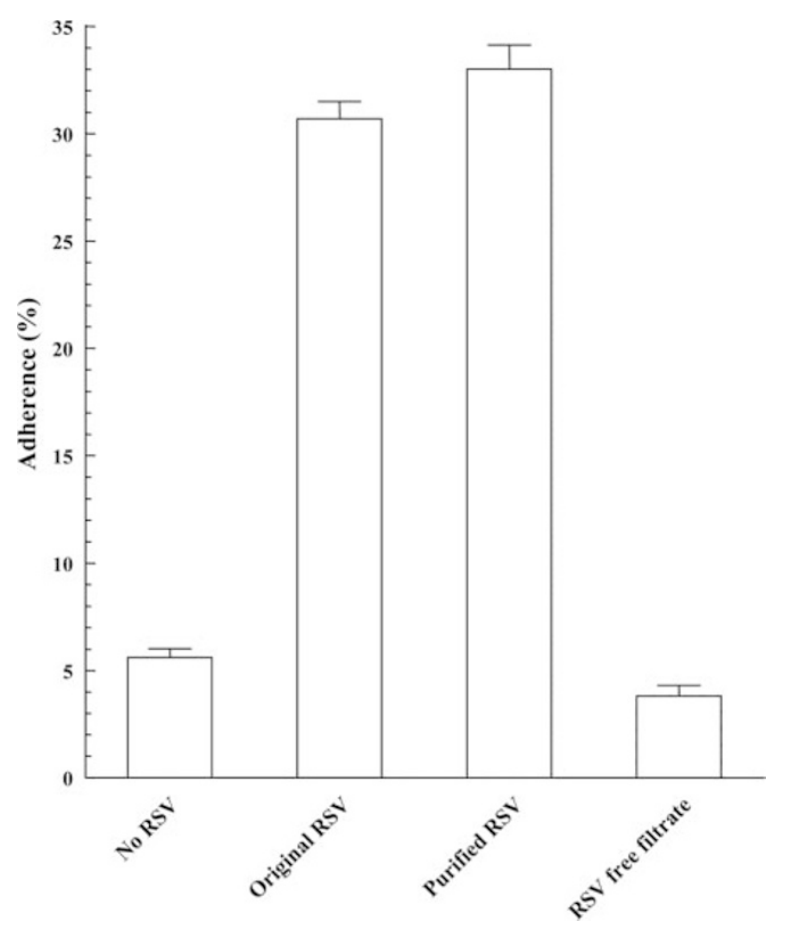

Figure 4. Original and PEG-precipitated, purified RSV suspension gave similar increased adherence of strain Pa01 to IB3-1 monolayer compared with control, whereas there was no increase of adherence with RSV free filtrate. Data from one of two representative experiments \pm SD performed in duplicate are shown.

\section{DISCUSSION}

Our study shows that both RSV infection of human respiratory epithelial cells and simultaneous addition of coincubated RSV and $P$. aeruginosa to noninfected epithelial cells strongly enhance the adherence of $P$. aeruginosa to these cells. This was shown with both laboratory and clinical bacterial strains and in multiple cell types. In addition, we observed direct binding of RSV to $P$. aeruginosa, suggesting that RSV possibly acts as a coupling agent between $P$. aeruginosa and epithelial cells. Specific blocking with heparin eliminates the increased adherence of $P$. aeruginosa, suggesting that this

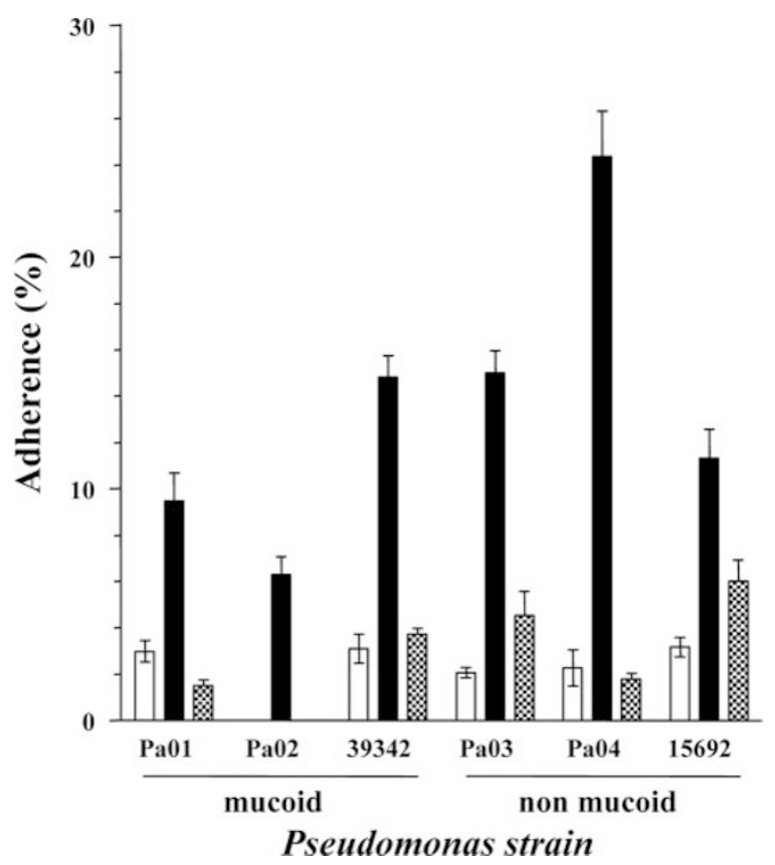

Figure 5. Pretreatment of IB3-1 cells with heparin (blocked bars) gave nearly complete blocking of the enhanced adherence of co-incubated RSV-P. aeruginosa complex (solid bars), when compared with $P$. aeruginosa alone (open bars) and as measured by cytometric fluorescence assay. This increase is significant for all strains $(t$ test, $p<0.05$ ). Data are represented as mean \pm SEM of three experiments; in each experiment seven wells per treatment were used. 

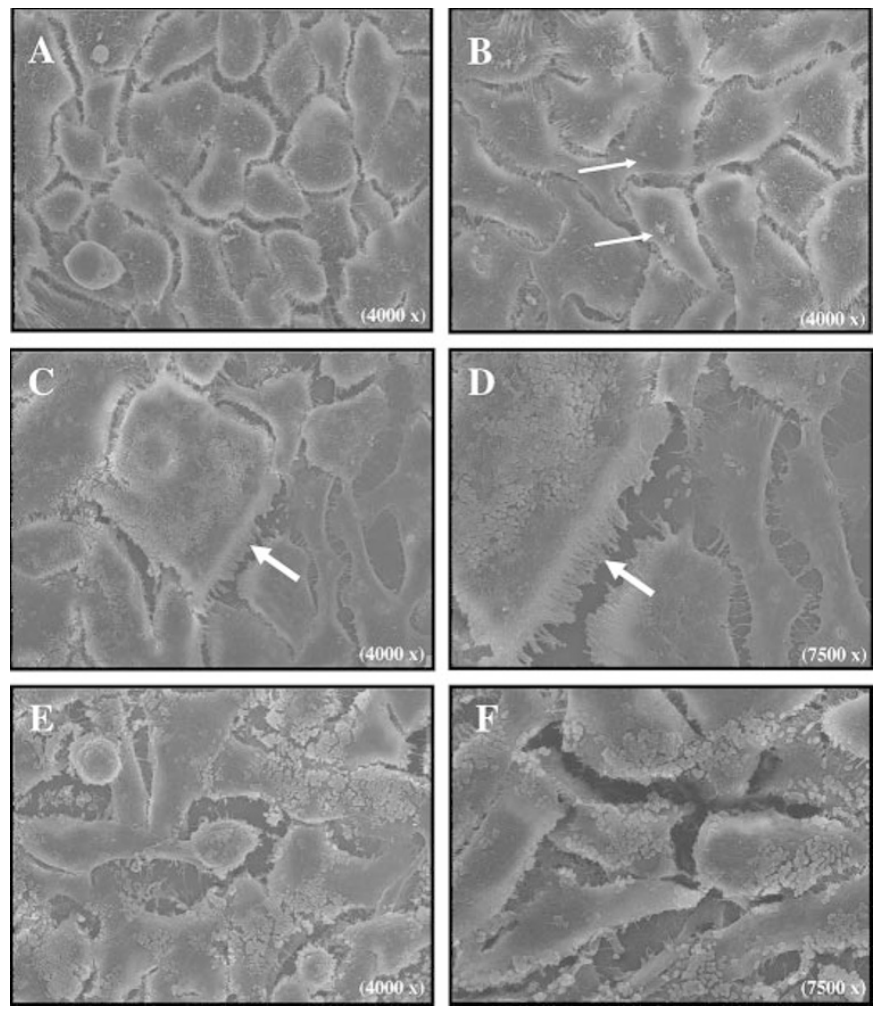

Figure 6. Visualization of pseudomonal adherence to HEp-2 cells by scanning electron microscopy $(\times 4000 / 7500)$ shows noninfected cells without $P$. aeruginosa $(A, \times 4000)$, noninfected cells with little adherence (arrows) of $P$. aeruginosa ( $B, \times 4000)$, large amounts of $P$. aeruginosa mainly adhering to syncytia (arrows) of RSV infected cells $(C, \times 4000 ; D, \times 7500)$ and large amounts of $P$. aeruginosa adhering more evenly after simultaneous addition of co-incubated RSV and $P$. aeruginosa to noninfected cells $(E, \times 4000 ; F, \times 7500)$.

coupling of $P$. aeruginosa to the cells is mediated through RSV glycoprotein G. Scanning electron microscopy shows a striking difference in pattern of pseudomonal adherence to RSV-infected epithelial cells compared with simultaneous addition of co-incubated RSV and $P$. aeruginosa to noninfected cells, suggesting that different mechanisms of increased adherence are possible.

There are several potential mechanisms to explain our findings of RSV-induced enhancement of pseudomonal adherence to respiratory epithelial cells. Bacteria can bind to virusinduced and up-regulated cellular membrane proteins, and to induced viral glycoproteins like glycoprotein F, G, and neuraminidase on the surface of infected cells (16). We show in addition that RSV possibly acts as a coupling agent for pseudomonal binding to respiratory epithelial cells.

Our data offer several arguments to suggest such a direct coupling mechanism. Simultaneous addition of co-incubated RSV and $P$. aeruginosa results in increased binding of bacteria to RSV-uninfected monolayers. During the simultaneous addition of RSV and $P$. aeruginosa, expression or upregulation of cellular or viral proteins on the epithelial cell membranes, or up-regulation of inflammatory mediators is less likely because of the relatively short time interval between adding of co-incubated bacteria and virions and the assessment of adherence. In addition, filtration experiments show that the enhancing effect of RSV on Pseudomonas adherence is due only to RSV virions and not to any other products in the RSV suspension, such as inflammatory mediators produced during RSV culture. We demonstrate furthermore a direct binding between RSV and $P$. aeruginosa in a dose-dependent manner.

We suggest that this coupling mechanism can be mediated through binding of $P$. aeruginosa to glycoprotein $\mathrm{G}$, known to be expressed on the surface of RSV (16). Pretreatment of respiratory cells with heparin, which interacts with glycoprotein $\mathrm{G}$ (17), gives a nearly complete blocking of the enhanced adherence of co-incubated RSV-P. aeruginosa complex. Nonspecific blocking by heparin, e.g. inhibition of inflammatory mediators, is less probable in view of the short time interval.

Scanning electron microscopy confirms the findings of the fluorescence assays by showing only little adherence of $P$. aeruginosa to respiratory epithelial cells in the absence of RSV, and a strongly enhanced pseudomonal adherence in the presence of the virus. It shows furthermore a striking difference in pattern of bacterial adherence. While $P$. aeruginosa mainly adheres to syncytia after RSV infection of epithelial cells, the strongly increased adherence is more evenly distributed with the simultaneous addition of RSV and $P$. aeruginosa to noninfected cells. This could suggest that the RSV-P. aeruginosa complex can bind to any epithelial cell, whereas adherence of $P$. aeruginosa alone is enhanced by binding, presumably to virus-induced glycoproteins expressed on syncytia after RSV infection.

The present study is the first to report that RSV infection can increase the adherence of $P$. aeruginosa to respiratory epithelial cells in vitro. This corresponds to earlier clinical observations that initial Pseudomonas colonization might be facilitated by respiratory viral infections, especially by RSV (12-14). In addition, a viral infection can be associated with a rise in antipseudomonal antibodies in CF patients with intermittent or chronic pseudomonal colonization, especially in case of RSV infection (14). These findings are furthermore consistent with several studies that demonstrate enhanced adherence of bacteria, like Haemophilus influenzae and Staphylococcus aureus, to epithelial cells after viral infection in vitro $(9,18,19)$ and in vivo $(20,21)$. However, there are only few experimental data about synergism between viruses and $P$. aeruginosa in inducing infections. In a study by Ramphal $e t$ al. (26), $P$. aeruginosa only adheres to murine tracheas when injured by influenza infection. Seki et al. (27) showed influenza infection to play an important role in inducing fatal pneumococcal pneumonia in chronic $P$. aeruginosa-infected mice. Recently, Stark et al. (24) found a decreased clearance of $P$. aeruginosa from the lungs after a RSV infection in non-CF mice. Although $P$. aeruginosa is the most important bacterial pathogen in CF, the synergism between RSV and $P$. aeruginosa in infecting respiratory epithelial cells seemed not to be CF-specific in the present study. We observed no consistent differences between IB3-1 (CF) cells and HEp-2 or A549 (non-CF) cells, however, not having used isogenic cell lines.

There are limitations of our study. Our in vitro data do not necessarily reflect in vivo situations, and the role of other 
viruses on adherence of $P$. aeruginosa still has to be determined. However, we used multiple cell types and both clinical and laboratory bacterial strains with consistent findings of enhanced adherence. Adherence of $P$. aeruginosa without RSV varies in similar cell types between the different experiments, but monolayers were, depending on the experiment, grown to confluence in 2 or 3-4 d, respectively. Induction of specific bacterial factors might be another mechanism influencing adherence, but was outside the scope of the present study.

Our findings of RSV-induced enhancement of pseudomonal adherence in general might be interesting in a clinical perspective. $P$. aeruginosa is a selective and important pathogen in CF. Recent data in CF mice underline the relevance of RSV infections in pulmonary morbidity in CF (25). It might be suggested that viral infections like RSV facilitate bacterial colonization, e.g. with $P$. aeruginosa. Further knowledge about synergism between viruses and bacteria in CF lung disease might result in new therapeutic strategies to improve prognosis of patients with CF. If our in vitro results can be confirmed in vivo, the suggestion that viral infections can play a role in bacterial colonization and pulmonary CF exacerbations might implicate adaptation of current therapeutic strategies and development of new options. For example, nebulization with heparin might possibly reduce the risk of bacterial colonization and infection in case of an RSV infection. Moreover, prevention of viral infections will be emphasized, e.g. by active or passive immunization. Viral inhibitors might be used in an early phase of a viral infection, antibiotic treatment, or prophylaxis during a viral infection, and development of other specific viral-bacterial interaction blockers might provide interesting options.

In summary, this is the first report that RSV infection of respiratory epithelial cells and simultaneous addition of RSV and $P$. aeruginosa to noninfected epithelial cells both strongly enhance the in vitro adherence of $P$. aeruginosa. In this process, RSV possibly acts as a coupling agent. Heparin seems able to block RSV and to eliminate this enhanced bacterial adherence. It confirms the suggestion that viral infections possibly play a role in bacterial colonization and pulmonary $\mathrm{CF}$ exacerbations. If these results can be confirmed in vivo, it could have important implications on treatment of CF pulmonary disease.

Acknowledgments. The authors thank W.A.M. van Maurik from Electron Microscopy Utrecht, Utrecht University, for technical assistance in performing scanning electron microscopy studies.

\section{REFERENCES}

1. Gibson RL, Burns JL, Ramsey BW 2003 Pathophysiology and management of pulmonary infections in cystic fibrosis. Am J Respir Crit Care Med 168:918-951
2. Sadikot RT, Blackwell TS, Christman JW, Prince AS 2005 Pathogen-host interactions in Pseudomonas aeruginosa pneumonia. Am J Respir Crit Care Med 171:1209-1223

3. Hiatt PW, Grace SC, Kozinetz CA, Raboudi SH, Treece DG, Taber LH, Piedra PA 1999 Effects of viral lower respiratory tract infection on lung function in infants with cystic fibrosis. Pediatrics 103:619-626

4. Wang EE, Prober CG, Manson B, Corey M, Levison H 1984 Association of respiratory viral infections with pulmonary deterioration in patients with cystic fibrosis. N Engl J Med 311:1653-1658

5. Armstrong D, Grimwood K, Carlin JB, Carzino R, Hull J, Olinsky A, Phelan PD 1998 Severe viral respiratory infections in infants with cystic fibrosis. Pediatr Pulmonol 26:371-379

6. Smyth AR, Smyth RL, Tong CY, Hart CA, Heaf DP 1995 Effect of respiratory virus infections including rhinovirus on clinical status in cystic fibrosis. Arch Dis Child 73:117-120

7. Hakansson A, Kidd A, Wadell G, Sabharwal H, Svanborg C 1994 Adenovirus infection enhances in vitro adherence of Streptococcus pneumoniae. Infect Immun 62:2707-2714

8. Ishizuka S, Yamaya M, Suzuki T, Takahashi H, Ida S, Sasaki T, Inoue D, Sekizawa K, Nishimura H, Sasaki H 2003 Effects of rhinovirus infection on the adherence of Streptococcus pneumoniae to cultured human airway epithelial cells. J Infect Dis 188:1928-1939

9. Jiang Z, Nagata N, Molina E, Bakaletz LO, Hawkins H, Patel JA 1999 Fimbriamediated enhanced attachment of nontypeable Haemophilus influenzae to respiratory syncytial virus-infected respiratory epithelial cells. Infect Immun 67:187192

10. Okamoto S, Kawabata S, Nakagawa I, Okuno Y, Goto T, Sano K, Hamada S 2003 Influenza A virus-infected hosts boost an invasive type of Streptococcus pyogenes infection in mice. J Virol 77:4104-4112

11. Okamoto S, Kawabata S, Terao Y, Fujitaka H, Okuno Y, Hamada S 2004 The Streptococcus pyogenes capsule is required for adhesion of bacteria to virus-infected alveolar epithelial cells and lethal bacterial-viral superinfection. Infect Immun 72:6068-6075

12. Collinson J, Nicholson KG, Cancio E, Ashman J, Ireland DC, Hammersley V, Kent J, O'Callaghan C 1996 Effects of upper respiratory tract infections in patients with cystic fibrosis. Thorax 51:1115-1122

13. Johansen HK, Hoiby N 1992 Seasonal onset of initial colonisation and chronic infection with Pseudomonas aeruginosa in patients with cystic fibrosis in Denmark. Thorax 47:109-111

14. Petersen NT, Hoiby N, Mordhorst CH, Lind K, Flensborg EW, Bruun B 1981 Respiratory infections in cystic fibrosis patients caused by virus, chlamydia and mycoplasma-possible synergism with Pseudomonas aeruginosa. Acta Paediatr Scand 70:623-628

15. Hament JM, Aerts PC, Fleer A, Van Dijk H, Harmsen T, Kimpen JL, Wolfs TF 2004 Enhanced adherence of Streptococcus pneumoniae to human epithelial cells infected with respiratory syncytial virus. Pediatr Res 55:972-978

16. Hament JM, Kimpen JL, Fleer A, Wolfs TF 1999 Respiratory viral infection predisposing for bacterial disease: a concise review. FEMS Immunol Med Microbiol 26:189-195

17. Bourgeois C, Bour JB, Lidholt K, Gauthray C, Pothier P 1998 Heparin-like structures on respiratory syncytial virus are involved in its infectivity in vitro. J Virol 72:7221-7227

18. Davison VE, Sanford BA 1981 Adherence of staphylococcus aureus to influenza A virus-infected Madin-Darby canine kidney cell cultures. Infect Immun 32:118-126

19. Saadi AT, Blackwell CC, Raza MW, James VS, Stewart J, Elton RA, Weir DM 1993 Factors enhancing adherence of toxigenic Staphylococcus aureus to epithelial cells and their possible role in sudden infant death syndrome. Epidemiol infect 110:507517

20. Patel J, Faden H, Sharma S, Ogra PL 1992 Effect of respiratory syncytial virus on adherence, colonization and immunity of non-typable Haemophilus influenzae: implications for otitis media. Int J Pediatr Otorhinolaryngol 23:15-23

21. Sanford BA, Ramsay MA 1987 Bacterial adherence to the upper respiratory tract of ferrets infected with influenza A virus. Proc Soc Exp Biol Med 185:120-128

22. Ramphal R, Small PM, Shands JW Jr, Fischlschweiger W, Small PA Jr 1980 Adherence of Pseudomonas aeruginosa to tracheal cells injured by influenza infection or by endotracheal intubation. Infect Immun 27:614-619

23. Seki M, Higashiyama Y, Tomono K, Yanagihara K, Ohno H, Kaneko Y, Izumikawa K, Miyazaki Y, Hirakata Y, Mizuta Y, Tashiro T, Kohno S 2004 Acute infection with influenza virus enhances susceptibility to fatal pneumonia following Streptococcus pneumoniae infection in mice with chronic pulmonary colonization with Pseudomonas aeruginosa. Clin Exp Immunol 137:35-40

24. Stark JM, Stark MA, Colasurdo GN, LeVine AM 2006 Decreased bacterial clearance from the lungs of mice following primary respiratory syncytial virus infection. J Med Virol 78:829-838

25. Colasurdo GN, Fullmer JJ, Elidemir O, Atkins C, Khan AM, Stark JM 2006 Respiratory syncytial virus infection in a murine model of cystic fibrosis. J Med Virol 78:651-658 